\title{
Characterizing patients initiating abaloparatide, teriparatide, or denosumab in a real-world setting: a US linked claims and EMR database analysis
}

\author{
E.A. Imel ${ }^{1}$ (D) K. Starzyk ${ }^{2}$ (D) R. Gliklich ${ }^{2}$ (D) R.J. Weiss ${ }^{3}$ (D) Y. Wang ${ }^{3}$ (D) S.A. Williams ${ }^{3}$ (D)
}

Received: 11 October 2019 / Accepted: 11 March 2020 / Published online: 21 July 2020

(C) The Author(s) 2020

\begin{abstract}
Summary We characterized patients initiating abaloparatide (ABL), teriparatide (TPTD), or denosumab (DMAB) in a real-world clinical setting from a large medical and pharmacy claims database. Differences were noted in sex, age, pathologic fractures, comorbidity index, and prior bisphosphonate use for patients initiating ABL and TPTD compared with those receiving DMAB. Introduction To characterize patients initiating abaloparatide (ABL), teriparatide (TPTD), or denosumab (DMAB) treatment in a real-world clinical setting.

Methods Patients aged $\geq 18$ years initiating ABL, TPTD, or DMAB between May 1, 2017, and September 24 , 2018 (without receiving the same drug in the previous 12 months), were identified using the OM1 Data Cloud, which contains medical and pharmacy claims from approximately 200 million US patients. The index date was the date of initial prescription or dispensing for ABL, TPTD, or DMAB during the study period.

Results During the study period, 2666 patients initiated ABL, 9210 TPTD, and 116,718 DMAB. Mean age (standard deviation) was 69.2 (10.6) years for the ABL cohort, 68.6 (11.3) for TPTD, and 72.1 (10.2) for DMAB $(P<0.001$; ABL vs DMAB). Proportionally more patients initiating ABL were female $(95.2 \%$ ABL, 86.9\% TPTD, and 91.3\% DMAB, $P<0.001$ ABL vs TPTD or DMAB). Nearly twice as many patients initiating ABL (19.1\%) and TPTD (18.8\%) had a previous pathologic/fragility fracture vs DMAB $(9.6 \% ; P<0.001$ ABL vs DMAB). Fewer patients initiating ABL (36.3\%) or TPTD (39.7\%) had Charlson comorbidity index of $\geq 2$ vs DMAB $(48.4 \% ; P<0.001$ ABL vs DMAB). Before initiating ABL, TPTD, or DMAB, 44.3\%, $33.8 \%$, and $33.9 \%$ of patients had prior osteoporosis treatment, respectively. Bisphosphonate use was more common before initiating ABL (19.2\%) or TPTD (19.6\%), than before initiating DMAB $(16.6 \% ; P<0.001$ ABL vs DMAB).

Conclusions Patients initiating ABL and TPTD differed in sex, age, pathologic fractures, comorbidity index, and prior bisphosphonate use compared with those initiating DMAB.
\end{abstract}

Keywords Abaloparatide $\cdot$ Denosumab · Osteoporosis $\cdot$ Patient characteristics $\cdot$ Real-world $\cdot$ Teriparatide

Electronic supplementary material The online version of this article (https://doi.org/10.1007/s00198-020-05388-y) contains supplementary material, which is available to authorized users.

E.A. Imel

eimel@iu.edu

1 Department of Medicine, Endocrinology, Indiana University School of Medicine, Gatch Hall, Suite 380 F, 1120 W. Michigan St., Indianapolis, IN 46202-5111, USA

2 OM1, Inc., 800 Boylston St, Boston, MA 02199, USA

3 Radius Health, Inc., 950 Winter St, Waltham, MA 02451, USA

\section{Introduction}

Emerging evidence supports sequential therapy with an anabolic agent followed by an antiresorptive agent as an effective treatment option for postmenopausal women with osteoporosis at high risk for fractures $[1,2]$. However, payors often dictate starting with less expensive agents and only advancing to more expensive agents if there is a contraindication, intolerance, or failure of the less expensive agents. Antiresorptive treatments, such as bisphosphonates and denosumab (DMAB), are frequently used for the treatment of postmenopausal osteoporosis. While antiresorptive agents improve bone mineral density (BMD) by reducing bone remodeling and increasing the degree of mineralization, anabolic agents 
increase BMD and bone quality, leading to improvements in bone strength [3-6].

Teriparatide (TPTD) is a parathyroid hormone analog (PTH1-34) and abaloparatide (ABL) is a parathyroid hormone (PTH)-related peptide analog. Each increases BMD through anabolic effects that increase bone formation [7-9]. Both ABL and TPTD have evidence of fracture risk reduction in postmenopausal osteoporosis $[8,9]$ and, like DMAB, are FDA approved for treatment of postmenopausal osteoporosis with high risk for fractures (TPTD approved November 26, 2002, and ABL April 28, 2017) [10-12], defined as having a history of osteoporosis-related fractures, multiple risk factors, or having failed other osteoporosis therapies $[2,11]$. DMAB has been approved for the treatment of osteoporosis in men, and both TPTD and DMAB are indicated for the treatment of osteoporosis related to sustained systemic glucocorticoid therapy in men and women. All three medications are administered subcutaneously, once daily for ABL and TPTD and every 6 months for DMAB. Use of ABL or TPTD for more than 2 years during a patient's lifetime is not recommended.

Risk-sharing contracts between payors and manufacturers require data analysis to establish the relative benefits and risks of various medications. Clinical treatment choices are impacted by multiple factors. Understanding characteristics of real world treated patients is the first step toward effectiveness evaluation especially in this disease area where treatment history and fracture history have an important impact on treatment response. Previous studies reported pretreatment differences in patients who initiated TPTD versus bisphosphonates or other osteoporosis therapies [13, 14]; however, no data are available in the current literature regarding characteristics of patients initiating $\mathrm{ABL}$ versus other treatment options. Characterizing patients initiating $\mathrm{ABL}$ vs those initiating TPTD or DMAB will enable a better understanding of treatment selection and allow for proper casemix adjustment for future real-world effectiveness and comparative effectiveness studies. Collecting enough meaningful data through traditional disease registries can be time consuming, and most importantly because enrollment is unpredictable, data collection can be difficult; however, real-world data, such as electronic medical and pharmacy claims databases, can track larger numbers of patients and outcomes more efficiently over time.

This report examines the characteristics of patients initiating treatment with ABL compared with TPTD or DMAB in the real-world clinical setting using a large national medical and pharmacy claims database.

\section{Methods}

\section{Data source}

The OM1 Data Cloud is a continuously updated, deterministically linked, multi-source US-based dataset that includes payor-agnostic medical claims that contain billing and coding history on inpatient and outpatient encounters from acute care facilities, ambulatory surgery centers and clinics, and problem lists, and pharmacy claims for prescription filling or medication dispensing for over 200 million US-based patients [15-17]. Data are ingested, cleaned, and normalized while being updated to create longitudinal patient journeys for analysis. Data checks for range and consistency are used to improve accuracy of the data. Data completeness is dependent on the individual variable and how it is measured and recorded either during routine practice or in administrative data sources.

For a subgroup of patients (over 35 million), linked clinical details from electronic medical records (EMRs) were also included to augment diagnosis and procedure codes and to capture variables not readily available from claims such as smoking history, alcohol use disorder, and most recent body mass index (BMI). As EMR data are only available for a subgroup of patients, limited data are reported on variables that are typically available only through EMRs.

\section{Study population}

Eligible patients were those $\geq 18$ years of age initiating ABL, TPTD, or DMAB. Although osteoporosis is most common in postmenopausal women [2], 18 years was used as the age cutoff to allow for inclusion of patients being treated for secondary osteoporosis. The index date was defined as the first occurrence of either a prescription or dispensing of medication for ABL, TPTD, or DMAB. For evaluation of treatment patterns, the date when first dispensed was used. The identification period for patient characterization was between May 1, 2017 (coinciding with the FDA approval of ABL), and September 24, 2018. An exploratory analysis of treatment patterns was also conducted between May 1, 2017, and May 7, 2018, for a subpopulation of patients with pharmacy claims data available for pre-index exposure to TPTD or DMAB.

Patients were required to have at least 12 months of data available prior to the index date and were excluded from a cohort (ABL, TPTD, or DMAB) if there was evidence of treatment with the same drug in the 12 months prior to the index date. Patients were not required to have diagnosis codes for osteoporosis or osteopenia, so that we could capture the full prescribing patterns. Additional exclusion criteria were diagnosis codes for Paget's disease and any malignancy (except for nonmelanoma skin cancer).

\section{Demographic data}

Patient demographic characteristics, including age, sex, race, and insurance type (commercial, Medicare, Medicaid, other) were collected. 


\section{Medical diagnoses}

Medical diagnoses were assessed using the presence of ICD-9 and ICD-10 codes in diagnostic claims for inpatient or outpatient services regardless of the priority level of the code for the encounter. Osteoporosis disease history (i.e., diagnosis date and documented fractures) and osteoporosis-related treatment history (i.e., prior medication use) were assessed using structured (coded) data. Text-note-based clinical data was not available. Fractures were determined based on the presence of a diagnostic code for fracture at any position in the claim (primary, secondary, etc.) (Supplemental Table 1). Female patients who had a diagnosis code indicating menopause or postmenopausal status, had a history of bilateral oophorectomy or postprocedural ovarian failure, or were $\geq 55$ years at index date were considered postmenopausal.

The presence of procedure codes for osteoporosis diagnostic procedures within 12 months prior to or on the index date were recorded (BMD-dual energy X-ray absorptiometry [DXA] or BMD-other, e.g., quantitative computed tomography [QCT], ultrasound, or unspecified). Patients may have had codes for more than one procedure category. The resultant numerical measurement values are not available as part of this database.

Additional data on factors contributing to future fracture risk were collected from claims data and, for a subset of patients, from structured data within the EMR, and included codes for fracture, smoking history, alcohol use disorder, and the most recent body mass index (BMI) prior to the index date. Standard BMI categories for adults were utilized: underweight $\left(<18.5 \mathrm{~kg} / \mathrm{m}^{2}\right)$, normal $\left(18.5-24.9 \mathrm{~kg} / \mathrm{m}^{2}\right)$, overweight $(25.0$ $\left.29.9 \mathrm{~kg} / \mathrm{m}^{2}\right)$, and obese $\left(\geq 30 \mathrm{~kg} / \mathrm{m}^{2}\right)$. Tobacco use was identified by diagnosis codes indicating a history of tobacco use at any time prior to or including the index date including both current and former users. Alcohol use was only captured when codes for an alcohol use disorder occurred.

Additional comorbid conditions were detected using the presence of ICD-9 and ICD-10 codes, especially indicators supporting future fracture risk (Supplemental Table 2). Comorbidity burden was estimated using the Charlson comorbidity index (CCI) based on diagnosis codes documented on or prior to the index date [18].

\section{Treatment history}

Medical and pharmacy claims available for all patients in the OM1 database (either prescription documentation or medication dispensed) were used to assess cumulative duration of pre-index exposure for each cohort. Osteoporosis medication history included exposure to selected medications at any time prior to the index date. Patients could have been counted in multiple medication categories. Bisphosphonates included alendronate, zoledronic acid, ibandronate, risedronate, pamidronate, and etidronate. Selective estrogen receptor modulators (SERMs) included ospemifene and raloxifene. Other osteoporosis medications included ABL, TPTD, DMAB, and estrogen containing hormone replacement therapy. Xgeva, denosumab given every 4 weeks, is indicated for malignancies. As such, it was excluded using the National Drug Code and the Common Procedure Coding system. Calcitonin exposure was not included. Evidence of glucocorticoid use was assessed, stratified by route of administration (oral, inhaled, and nasal), at any time prior to the index date. Chronic oral glucocorticoid use was determined by evidence of at least 90 consecutive days of oral glucocorticoid use at any time prior to the index date.

\section{Sub-analysis: pre-index exposure to DMAB or TPTD}

In a subpopulation of patients, pharmacy claims data between May 1, 2017, and May 7, 2018, was used to quantify the extent of prior (pre-index) exposure to DMAB or TPTD in the treatment groups. For this sub-analysis, exposure was defined as having filled a prescription (medication dispensed). For purposes of calculating duration of DMAB exposure, a single injection of DMAB represented 6 months of exposure and discontinuation of DMAB was counted when a patient initiated another osteoporosis-specific medication or if they did not have a subsequent DMAB exposure within 60 days following the end of the prior exposure, whichever was earlier. This allowed for a grace period in assessing discontinuation for delays due to missed appointments, insurance approval issues, or other situations where drug treatment was actually being continued. Mean duration of therapy and the duration between pre-index treatment discontinuation and the start of index treatment were calculated in months.

\section{Statistical analyses}

Analyses were provided overall and by index medication. Patient characteristics, treatments, and comorbidities were summarized with descriptive statistics. Missing values were not imputed, as is typical for claims-based studies [19]. Statistical tests comparing groups of interest were based on analysis of variance (ANOVA) or chi-square test as appropriate. If significant differences were detected among the groups based on the global $P$ values, pairwise comparisons were performed, including $P$ values, differences between means and associated $95 \%$ confidence intervals, or risk differences (i.e., difference between proportions) and associated $95 \%$ confidence intervals.

$P$ value was considered significant if $<0.05$. Statistical analyses were performed with SAS (version 9.4, Cary, NC). 


\section{Results}

\section{Demographic data}

Between May 1, 2017, and September 24, 2018, 2666 patients initiated ABL therapy, 9210 initiated TPTD therapy, and 116,718 initiated DMAB therapy (Fig. 1). The median duration of pre-index historical data per patient was 4.59 years (minimum 4.07, maximum 5.02), and the majority of patients were female $(91.1 \%)$ with a mean age (standard deviation [SD]) of 71.8 (10.4) years. Most patients $(76.3 \%)$ were $\geq 65$ years of age, $21.7 \%$ were $50-64$ years, and $1.9 \%$ were $\leq 49$ years. The majority of patients $(98.1 \%)$ were $\geq 50$ years of age and postmenopausal women $(86.8 \%)$.

Mean age (SD) for the ABL, TPTD, and DMAB cohorts, respectively, was 69.2 (10.6), 68.6 (11.3), and 72.1 (10.2) $(P<0.001$ for ABL vs DMAB) (Table 1). Although the majority of patients were female in all groups $(95.2 \% \mathrm{ABL}$, 86.9\% TPTD, and 91.3\% DMAB), the proportion of female patients was higher for patients initiating ABL vs both TPTD and DMAB (each $P<0.001$ ). Proportionally fewer patients initiating ABL or TPTD had commercial insurance and a higher proportion had Medicare compared with those initiating DMAB $(P<0.001)$ (Table 1$)$.

\section{Osteoporosis and related codes}

The mean duration in years from earliest detected osteoporosis diagnosis code to the index date was 3.0 (1.6) years overall and 2.8 (1.8), 2.3 (1.8), and 3.1 (1.6) for the ABL, TPTD, and DMAB cohorts, respectively (Table 2). Overall, $24.9 \%$ of patients had procedure codes for BMD-DXA detectable in the 12 months prior to the index date. Of patients initiating $\mathrm{ABL}$, fewer had BMD-DXA codes compared with patients initiating DMAB $(20.8 \%$ vs $25.5 \%, P<0.001)$, but more had BMD-
DXA performed within 12 months prior to index date compared with patients initiating TPTD $(20.8 \%$ vs. $18.6 \%$, $P=0.009$ ). Only $0.2 \%$ of patients in each treatment group had a procedure code for other types of BMD measurements (QCT, ultrasound, or unspecified).

Overall, $28.2 \%$ of patients had any prior fracture. Patients initiating $\mathrm{ABL}$ were more likely to have a history of any fracture $(43.7 \%)$ than patients initiating DMAB $(28.8 \%)$, including any vertebral fracture $(22.2 \%$ vs $10.9 \%, P<0.001)$, any nonvertebral fracture $(24.3 \%$ vs $18.1 \% P<0.001)$, any pathologic or fragility fracture $(19.1 \%$ vs $9.6 \%, P<0.001)$, or pathologic fracture in the year prior to index treatment initiation $(15.8 \%$ vs $6.7 \%, P<0.001)$ (Table 2). Fracture history was similar for patients initiating $\mathrm{ABL}$ and patients initiating TPTD, except patients initiating TPTD had more nonvertebral fractures $(27.3 \%$ vs $24.3 \%, P=0.002)$, including more hip (11.4\% vs $9.5 \%, P=0.006)$ and rib/sternal $(3.8 \%$ vs $2.7 \%$, $P=0.011)$ fractures. Additional risk factors for fracture included tobacco use codes in $16.8 \%$ of patients $(18.7 \% \mathrm{ABL}$, $20.4 \%$ TPTD $16.4 \%$ DMAB) and alcohol use disorder codes in $1.8 \%$ of patients $(2.1 \% \mathrm{ABL}, 2.6 \%$ TPTD, $1.7 \%$ DMAB). In patients for whom BMI data was available $(N=54,332)$, $5.1 \%$ were underweight $(6.4 \% \mathrm{ABL}, 6.3 \%$ TPTD, $4.9 \%$ DMAB), $45.2 \%$ had normal BMI $(46.8 \%$ ABL, $44.6 \%$ TPTD, $45.3 \%$ DMAB), $29.1 \%$ were overweight (26.5\% ABL, 26.6\% TPTD, 29.4\% DMAB), and 20.6\% were obese (20.3\% ABL, 22.5\% TPTD, 20.4\% DMAB).

Overall, the most prevalent comorbid conditions detected were osteoarthritis $(48.6 \%)$, gastrointestinal (GI) disorders (47.1\%), type 2 diabetes (20.5\%), and respiratory diseases (chronic obstructive pulmonary disease $20.4 \%$ and asthma $12.8 \%$ ) (Table 3). Patients initiating ABL had fewer GI disorders $(42.6 \%)$ than patients initiating TPTD $(46.9 \%)$ or DMAB $(47.3 \%)(P<0.001)$. Both ABL- and TPTD-treated patients had lower mean Charlson comorbidity index compared with
Fig. 1 Patient disposition. ABL, abaloparatide; DMAB, denosumab; TPTD, teriparatide

\section{OM1 Data Cloud}

(Study period May 1, 2017 to September 24, 2018)

\section{De-Identified, Healthcare and Pharmacy Claims Data \\ for $>200$ million patients (female and male), of whom approximately 187 million are aged $\geq 18$ years \\ - Claims data were linked to electronic medical records for $\sim 19 \%$ of patients (>35 million patients)}

$\geq 1$ new prescription for ABL, TPTD, or DMAB between May 1, 2017 and September 24, 2018

DMAB

116,718 patients 


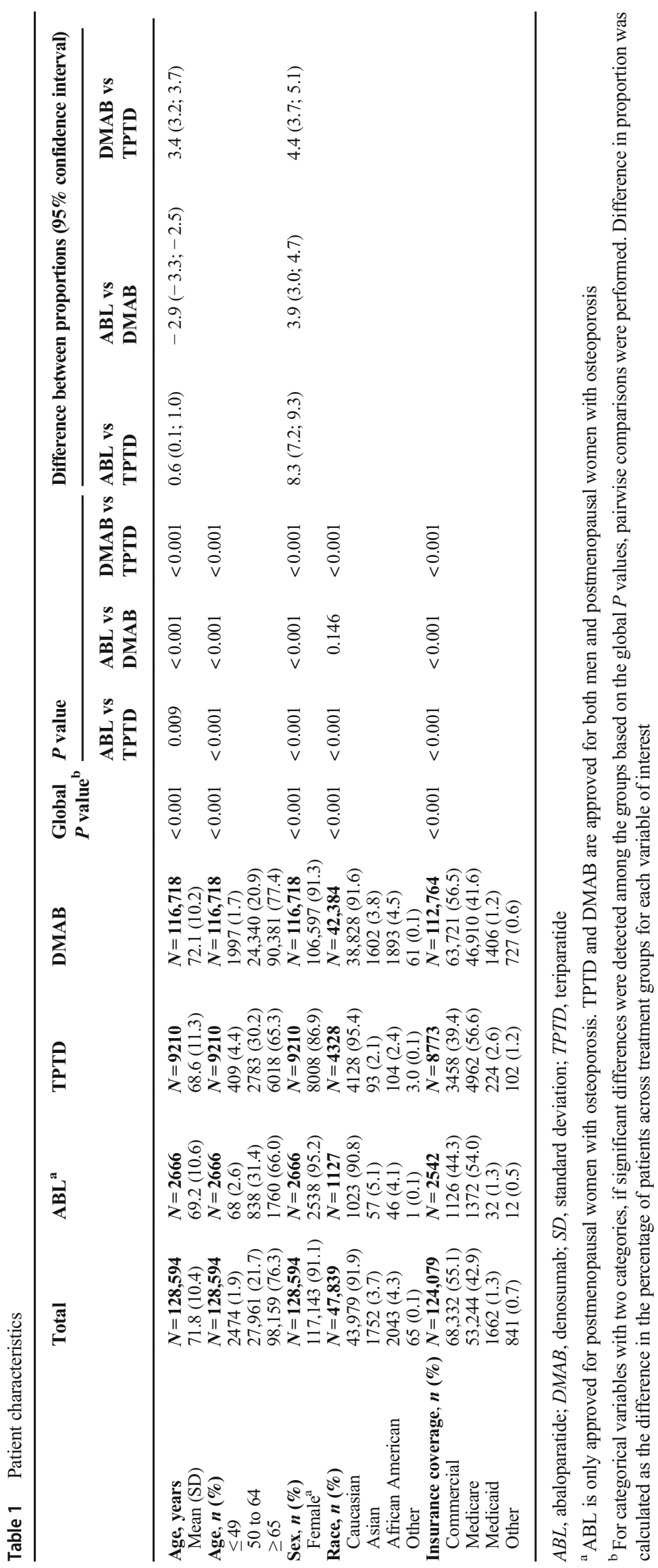




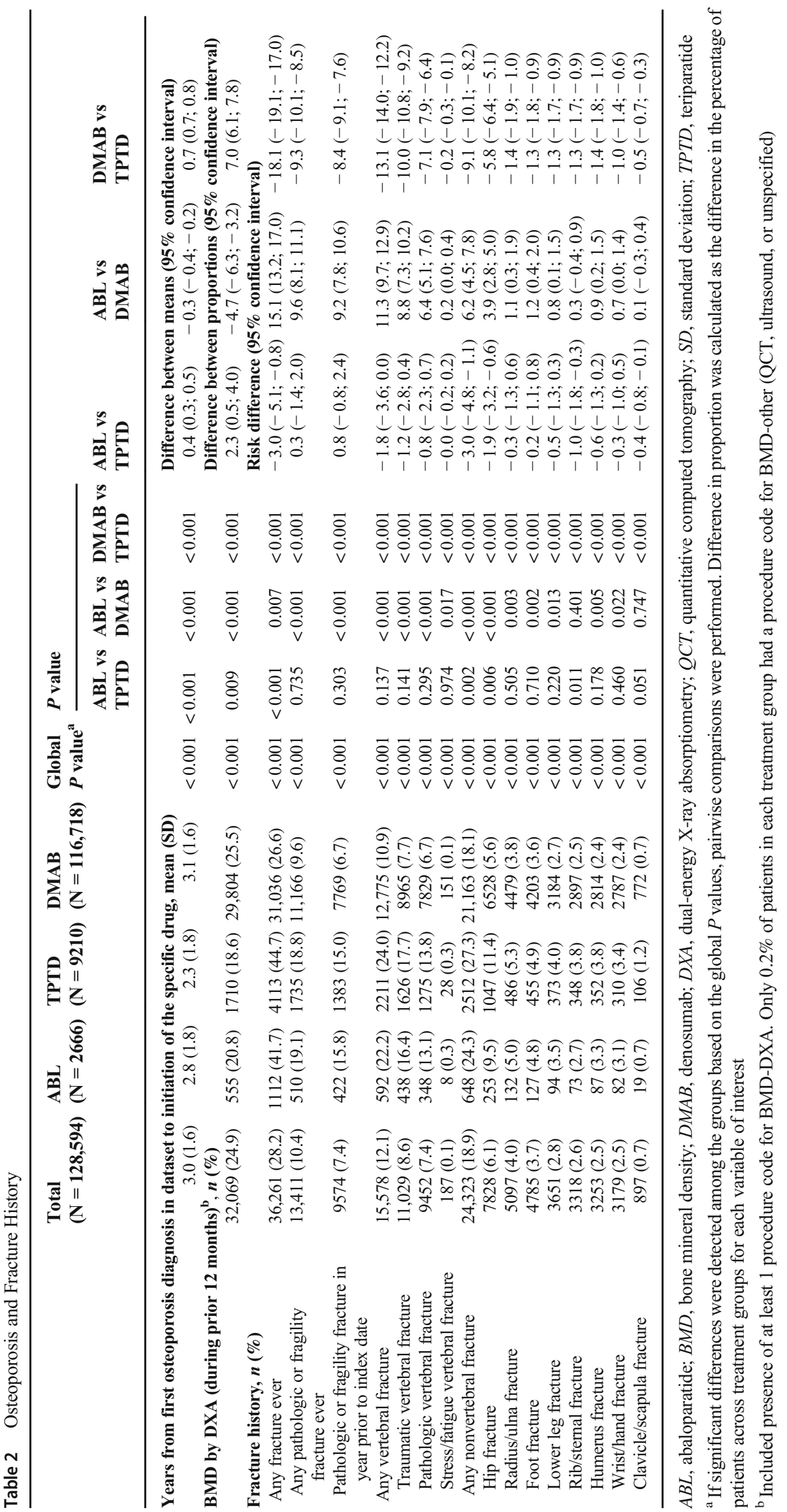


DMAB-treated patients, with $36.3 \%, 39.7 \%$, and $48.4 \%$ of ABL, TPTD, and DMAB patients, respectively, having a score of $\geq 2$ ( $P<0.001)$, suggesting greater comorbidity burden among patients chosen for DMAB.

Approximately $58 \%$ of all patients had one or more conditions associated with increased risk of fall, with visual impairment being the most frequent $(33.1 \%)$, followed by neuromuscular conditions $(23.3 \%)$, and dizziness/vertigo (17.3\%). The frequency was similar for all treatment groups (Table 3).

\section{Treatment history}

Pre-index data for treatment history are presented for all patients with any prescribing or claims data. Pre-index, $44.3 \%$, $33.8 \%$, and $33.9 \%$ of patients initiating ABL, TPTD, or DMAB had prior osteoporosis treatment, respectively. Specifically regarding pre-index therapy, $16.9 \%$ of patients received bisphosphonate(s), 1.9\% SERMs, and 20.3\% other osteoporosis medications. Alendronate was the most commonly used bisphosphonate, followed by zoledronic acid and ibandronate.

Rates of documented prior bisphosphonate use were similar in patients initiating ABL and TPTD and lower for patients initiating DMAB $(19.2 \%, 19.6 \%$, and $16.6 \%$, respectively; $P<0.001$ for ABL vs DMAB) (Table 4). Less than $5 \%$ of patients initiating ABL, TPTD, or DMAB were exposed to SERMs and $<10 \%$ had prior HRT. Prior DMAB use was more common for patients initiating ABL compared with patients initiating TPTD (14.8\% vs $7.1 \%, P<0.001)$. Prior TPTD use was more common for patients initiating ABL compared with patients initiating DMAB (14.7\% vs $1.9 \%$, $P<0.001)$.

Additionally, $47.4 \%$ of all patients had any exposure to glucocorticoids and $7.1 \%$ to long-term oral glucocorticoids (at least 90 consecutive days) prior to their index date, with $19.2 \%$ currently on glucocorticoids and $0.7 \%$ currently on long-term oral glucocorticoids. Exposure to any glucocorticoids was similar for patients initiating $\mathrm{ABL}$ and patients initiating TPTD; however, chronic oral glucocorticoid use and current glucocorticoid use (any or chronic oral) were significantly higher in patients initiating TPTD $(P<0.001)$ (Table 4).

\section{Pre-index exposure to TPTD and DMAB in patients with pharmacy claims data available}

As pharmacy claims (medication dispensing) data provide more confident assessments of patients starting and continuing a medication, the subset of patients having pharmacy claims data with index dates between May 1, 2017, and May 7, 2018, were included in a subanalysis. Overall, 78,789 patients initiating ABL, TPTD, or DMAB had pharmacy claims data available (in addition to healthcare claims data).

Of patients initiating ABL, 204 and 166 had pre-index exposure to TPTD and DMAB, respectively. The mean duration (SD) of pre-index TPTD therapy was 3.6 (4.4) months and of pre-index DMAB therapy was 12.9 (10.4) months (Table 5). The mean time between TPTD discontinuation and ABL initiation was 6.5 (11.4) months and between DMAB discontinuation and ABL initiation was 11.4 (13.2) months. Data regarding the use of other osteoporosis medications during this interval was not collected. Of patients initiating TPTD, 255 had pre-index exposure to DMAB. The mean duration of pre-index DMAB therapy was 12.4 (9.7) months, and the mean time between DMAB discontinuation and TPTD start was 17.1 (14.3) months (Table 5). Of patients initiating DMAB, 766 had pre-index TPTD utilization. The mean duration (SD) of pre-index TPTD therapy was 6.8 (7.4) months, and the mean time between TPTD discontinuation and DMAB start was 26.5 (15.1) months.

\section{Discussion}

The current study is the first to our knowledge to compare clinical characteristics of patients initiating ABL to those initiating TPTD or DMAB in the real-world setting following the FDA approval of ABL in May 2017. Real-world data for TPTD and DMAB have been previously reported [13, 14, 20], while less data are available for the more recently approved ABL. Overall, the patients included in this analysis were diverse and representative of the US osteoporosis population. In this population, it appears that patients initiating anabolic therapies (ABL and TPTD) have more similar demographics and clinical characteristics, compared with patients initiating antiresorptive therapy with DMAB. The reasons for these differences are not known but may be due to access to therapy through payors, clinician interpretation of available evidence on safety or efficacy, patient willingness to take a daily injectable therapy, or patient or physician concerns regarding side effects. The higher proportion of females in patients initiating ABL likely reflects lack of an FDA indication for $\mathrm{ABL}$ for treatment of osteoporosis in men.

Patients initiating anabolic therapy were more likely to have a history of fractures, suggesting physicians may be reserving anabolic therapy for patients perceived at higher fracture risk. The relatively low proportion of patients overall who initiated treatment with a prior fracture in our study is consistent with previous studies indicating that 70 to $80 \%$ of patients who sustain a fracture are neither diagnosed nor treated for osteoporosis $[21,22]$. In addition some patients who had a fracture history may be initiating other osteoporosis therapies instead of those in this study. 


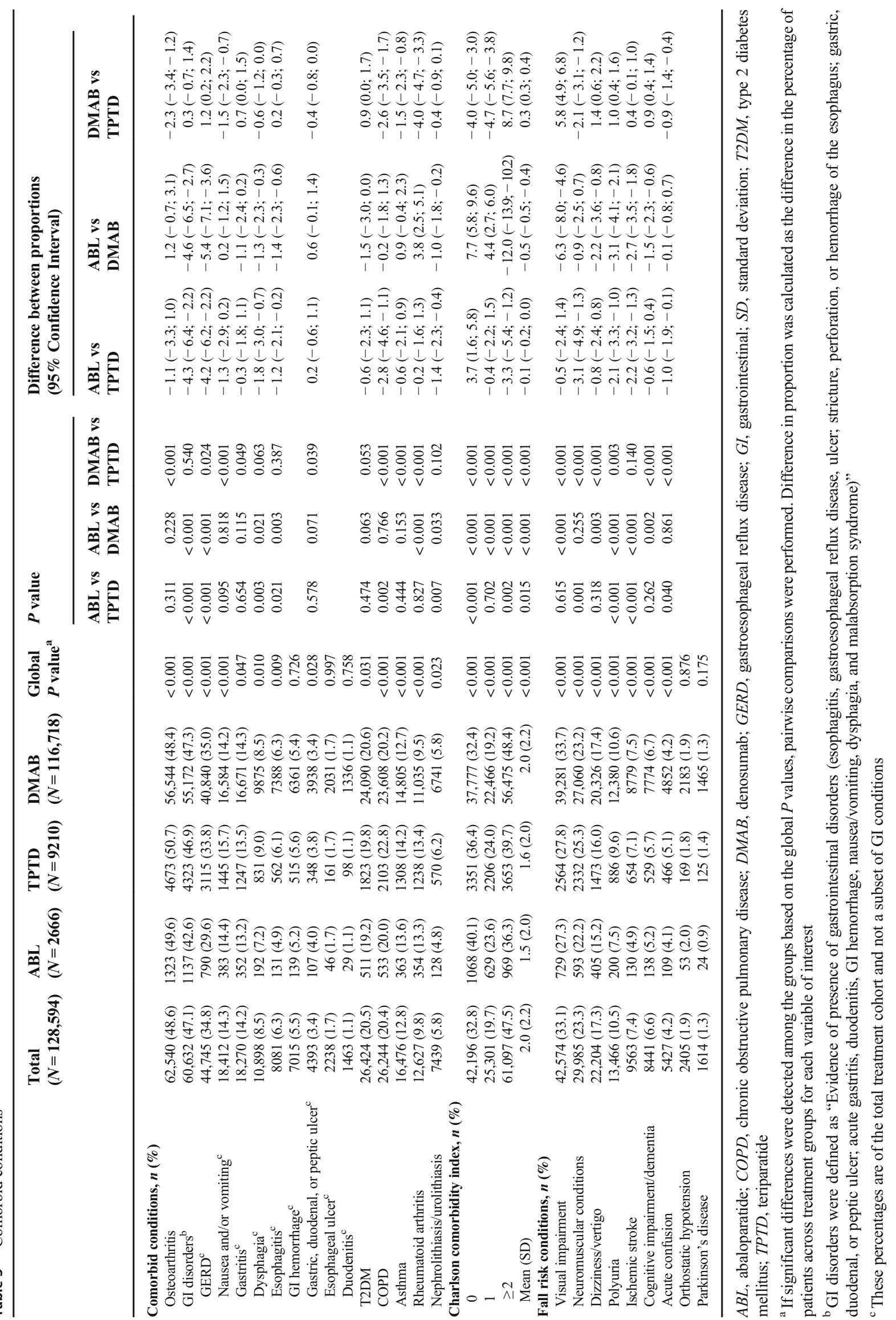




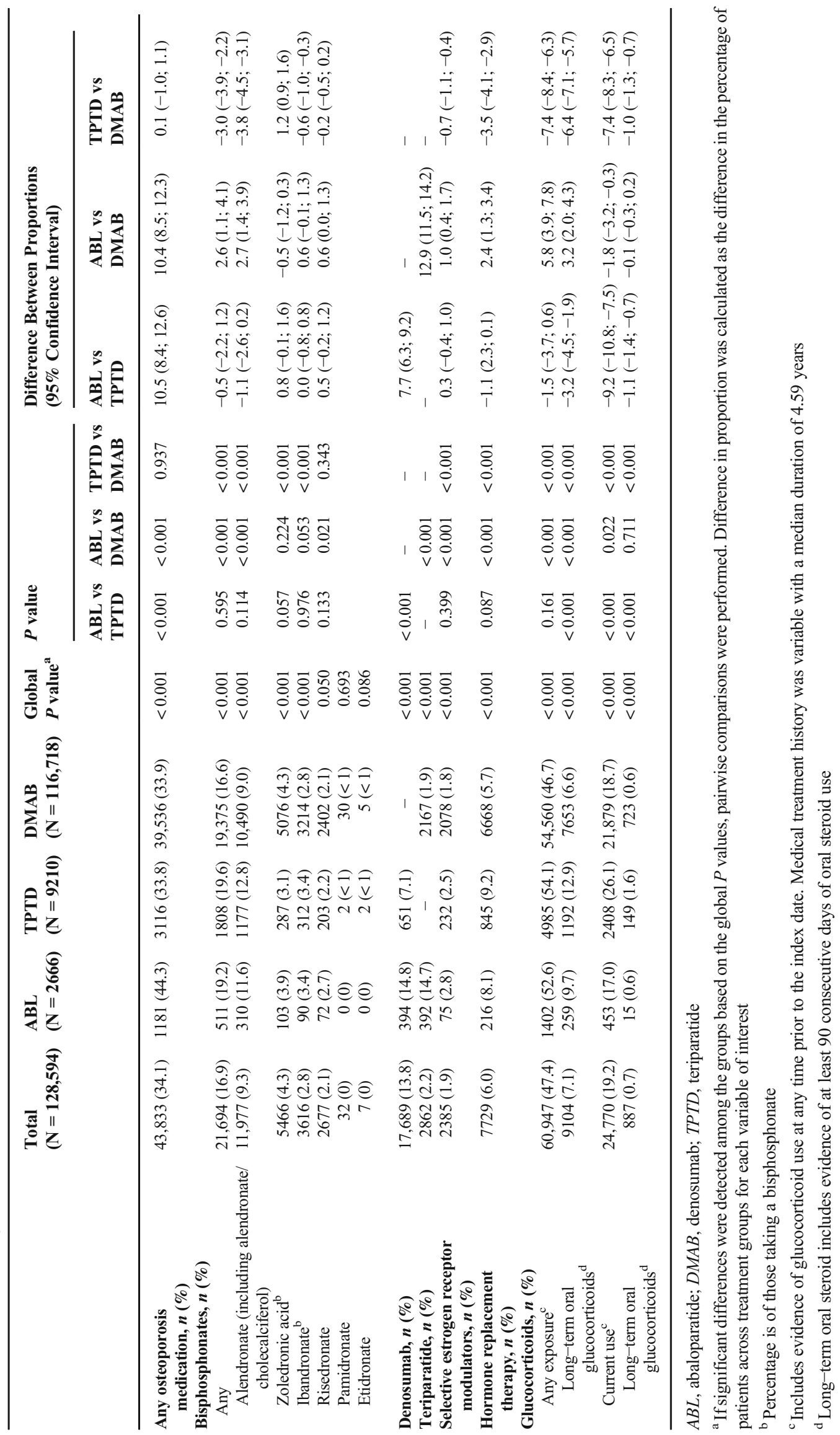


Two previous studies found that patients initiating an anabolic therapy (TPTD) had poorer health status and were more likely to have a history of fracture than patients initiating bisphosphonates or other osteoporosis treatments [13,14]. Patients initiating TPTD in prior studies were older than patients initiating other therapies, while patients initiating ABL or TPTD in our study were younger than those initiating DMAB. However, prior studies compared TPTD with bisphosphonates, raloxifene, nasal calcitonin, or HRT, whereas our study only compared ABL, TPTD, and DMAB. In our study patients receiving DMAB had worse health status suggested by higher Charlson comorbidity index than patients receiving ABL or TPTD.

The most prevalent comorbidities in our study included osteoarthritis and conditions often associated with poor bone quality or bone loss (i.e., diabetes and respiratory disorders) $[23,24]$. GI disorders were also highly prevalent $(47.1 \%)$ among patients selected for these injectable therapies, which may be due to concerns regarding reported GI side effects associated with oral bisphosphonate [23]. In a recent study of US managed care enrollees, patients with post-treatment GI diagnoses were $35.6 \%$ more likely to discontinue oral bisphosphonates or switch treatment $(\mathrm{HR}=1.356,95 \% \mathrm{CI}=$ 1.318-1.396) during the 12-month follow-up compared with those without post-treatment GI diagnoses [23]. Further, the high rate of glucocorticoid use in our population may warrant further evaluation due to potential impact on future fracture risk and to inform choice of treatment.

Also, of note, there were higher percentages of patients with rheumatoid arthritis in the ABL and TPTD treatment groups compared with the DMAB group. Although the current study did not collect provider specialty data, previous studies have shown that a higher proportion of patients treated by specialists are on anabolic agents compared with patients treated by primary care physicians [25].

A large portion of our population had no documentation of prior osteoporosis therapies during a median of 4.59 (minimum 4.07, maximum 5.02) years within this database prior to index date. Emerging evidence supports sequential therapy starting with anabolics in patients at high risk for fractures $[1,2]$. However, the current study suggests that in practice, the vast majority of patients are treated with antiresorptive agents first (counting bisphosphonates and DMAB, etc.) and anabolics are presumably reserved for those with perceived treatment failures or increasing risk factors, which may not be optimal. Our data also suggest that there may be a large delay (with means ranging from 6.5 to 26.5 months) after discontinuing DMAB or TPTD before initiation of subsequent therapy. This duration is concerning given the notable increased bone loss after discontinuing either agent and the high risk of vertebral fractures following DMAB treatment discontinuation [26]. Reasons for this delay in treatment are unclear but may be due to lack of proper documentation (e.g., uncaptured prescriptions) or healthcare provider knowledge of treatment guidelines, physician-led drug holidays (including misunderstanding of the drug mechanisms), patient tolerability issues, treatment costs, treatment failure, lack of adequate monitoring/follow-up, fear of side effects, or onset of new diseases. It is not known whether patients were prescribed other uncaptured osteoporosis treatments (apart from ABL, TPTD, DMAB) during this interval.

The results of the current study must be interpreted within the context of some limitations. First, one of the limitations of using secondary data in research is the lack of uniform availability of data components for all patients. In the current study, patients had a variable duration of health plan enrollment so complete treatment and medical codes were not available for all patients, which could alter the proportions of patients receiving any medical therapy. Nevertheless, there is no known imbalance regarding duration of health plan enrollment for patients by treatment cohort, and the median duration of pretreatment medical data available was similar between groups (4-5 years). Socio-economic data including patient co-pay and out-of-pocket costs were lacking, which could impact access to expensive treatments. Furthermore, we did not have the ability to differentiate type of Medicare coverage.

Another limitation is incomplete availability of data indicating disease severity or future fracture risk. While information on smoking and alcohol use disorders were included in the analyses, these data were based on available claims and EMR data and likely are under-representative of actual smoking and alcohol use disorder in the population studied. Further, only a subset of patients had integrated claims and EMR data, which would provide additional indicators of disease severity (i.e., BMI, race, etc.). Additionally, we do not have access to data on actual BMD results. Thus, we were unable to assess differences or changes in bone density as predictors of treatment choices.

Missing medical and treatment codes may lead to an underestimation of the reported diagnostic procedures (i.e., DXA). Notably reimbursements for these diagnostic tests are often every 2 years and osteoporosis diagnostic procedures were recorded only within 12 months prior to index date, which may limit how many patients were measured proximate to their initiation of our drugs of interest. It is possible that for some patients, bone density was assessed in a facility or location that was not captured in our data, or that the decision to start treatment was based on other risk factors such as fracture risk assessment tool (FRAX) score. While missing data due to administrative errors or encounters occurring outside the system are inherent to the secondary use of EMR data, there is no evidence that systematic bias between cohorts affected our results, which are generally consistent with other recent studies. In a previous study, of 8339 adherent patients on an oral bisphosphonate, only 3110 (37.3\%) had available DXA data within the 2-year pre-index period, and only 620 had pre- and post-index (13 to 36 months from the index date) DXA data [27]. In addition, Gillespie et al. reported that within 6 months 
Table 5 Pre-index exposure to TPTD and DMAB ${ }^{\mathrm{a}}$

\begin{tabular}{|c|c|c|c|}
\hline Pre-index exposures & $\begin{array}{l}\mathrm{ABL} \\
N=1814\end{array}$ & $\begin{array}{l}\text { TPTD } \\
N=5945\end{array}$ & $\begin{array}{l}\text { DMAB } \\
N=71,030\end{array}$ \\
\hline TPTD, $n$ & 204 & & 766 \\
\hline $\begin{array}{l}\text { Duration of therapy, months } \\
\text { Mean (SD) }\end{array}$ & $3.6(4.4)$ & & $6.8(7.4)$ \\
\hline $\begin{array}{l}\text { Interval between TPTD cessation and initiation of drug of interest, months }{ }^{b} \\
\text { Mean (SD) }\end{array}$ & $6.5(11.4)$ & & $26.5(15.1)$ \\
\hline $\mathrm{DMAB}, n$ & 166 & 255 & \\
\hline $\begin{array}{l}\text { Duration of therapy, months } \\
\text { Mean (SD) } \\
\text { Interval between DMAB cessation and initiation of drug of interest, months } \\
\text { Mean (SD) }\end{array}$ & $12.9(10.4)$ & $\begin{array}{l}12.4(9.7) \\
17.1(14.3)\end{array}$ & \\
\hline
\end{tabular}

$A B L$, abaloparatide; $D M A B$, denosumab; $S D$, standard deviation; $T P T D$, teriparatide

${ }^{a}$ Includes a subpopulation of patients between May 1, 2017, and May 7, 2018, for whom pharmacy claims data was available

${ }^{\mathrm{b}}$ Duration in months between pre-index treatment discontinuation and start of index treatment

of hip fracture, $13 \%$ of women underwent bone mass testing, $7 \%$ initiated pharmacotherapy, and only $3 \%$ did both [28].

Finally, this study only compared ABL, TPTD, and DMAB without comparing cohorts initiating other osteoporosis treatments. Comparisons of ABL vs TPTD and ABL vs DMAB were selected because real-world evidence on TPTD and DMAB have been previously reported, while less realworld data are available for the more recently approved ABL. Notably, prior to 2019, ABL and TPTD were the only anabolic agents FDA approved for postmenopausal osteoporosis at high risk for fragility fractures [10,11,29]; thus, we were not able to incorporate prescribing of other anabolic therapies (i.e., romosozumab) in our analysis.

Strengths of this study include the large sample size of patients initiating therapy identified based on claims data, suggesting the drug was actually obtained by patients. This large sample size allowed us to compare various clinical characteristics between groups. Future studies will allow us to compare real-world treatment effects on fractures, which are the most important outcomes for osteoporosis.

\section{Conclusions}

From this prospective registry we characterized patients initiating ABL, TPTD, and DMAB. The current study is the first to characterize patients initiating ABL in the USA in the year following FDA approval. The findings reflect similarities in patients' demographic and clinical characteristics for patients initiating the anabolic agents $A B L$ and TPTD, and notable differences compared with patients receiving DMAB. Data on treatment history suggest possible gaps in treatment following anabolic agents or DMAB, inconsistent with general recommendations regarding sequential treatment $[1,2]$, though further studies are required. Evaluations of treatment patterns and treatment order are of high importance in identification of factors contributing to health variations in health outcomes and costs of care. As data accumulate on real-world use of these treatments, future studies will pursue analysis of realworld treatment patterns, treatment persistence, and clinical outcomes during therapy which can be used to address stakeholders' need for comparative evidence.

Acknowledgments Funding for this study was provided by Radius Health, Inc. All listed authors meet the criteria for authorship set forth by the International Committee for Medical Journal Editors. Medical editorial support (Sarah Hummasti, PhD and Allyson Lehrman, DPM) and graphic services were provided by AOIC, LLC, and were funded by Radius Health, Inc.

Data availability The data for this study were obtained from the OM1 Data Cloud, a commercially available database maintained by OM1 (Boston, MA).

\section{Compliance with ethical standards}

Conflicts of interest EAI is a consultant for Radius Health, Inc. KS and RG are employees of OM1 and paid consultants for Radius Health, Inc. RJW, YW, and SAW are employees of and own equity stock in Radius Health, Inc.

Open Access This article is licensed under a Creative Commons Attribution-NonCommercial 4.0 International License, which permits any non-commercial use, sharing, adaptation, distribution and reproduction in any medium or format, as long as you give appropriate credit to the original author(s) and the source, provide a link to the Creative Commons license, and indicate if changes were made. The images or other third party material in this article are included in the article's Creative Commons license, unless indicated otherwise in a credit line to the material. If material is not included in the article's Creative Commons license and your intended use is not permitted by statutory regulation or exceeds the permitted use, you will need to obtain permission directly from the copyright holder. To view a copy of this license, visit http://creativecommons.org/licenses/by-nc/4.0/. 


\section{References}

1. Bone HG, Cosman F, Miller PD, Williams GC, Hattersley G, Hu MY, Fitzpatrick LA, Mitlak B, Papapoulos S, Rizzoli R, Dore RK, Bilezikian JP, Saag KG (2018) ACTIVExtend: 24 months of alendronate after 18 months of abaloparatide or placebo for postmenopausal osteoporosis. J Clin Endocrinol Metab 103(8):29492957

2. Camacho PM, Petak SM, Binkley N, Clarke BL, Harris ST, Hurley DL, Kleerekoper M, Lewiecki EM, Miller PD, Narula HS, PessahPollack R, Tangpricha V, Wimalawansa SJ, Watts NB (2016) American Association of Clinical Endocrinologists and American College of Endocrinology clinical practice guidelines for the diagnosis and treatment of postmenopausal osteoporosis - 2016executive summary. Endocr Pract 22(9):1111-1118

3. Boivin GY, Chavassieux PM, Santora AC, Yates J, Meunier PJ (2000) Alendronate increases bone strength by increasing the mean degree of mineralization of bone tissue in osteoporotic women. Bone 27(5):687-694

4. Dempster DW, Brown JP, Fahrleitner-Pammer A, Kendler D, Rizzo S, Valter I, Wagman RB, Yin X, Yue SV, Boivin G (2018) Effects of long-term denosumab on bone histomorphometry and mineralization in women with postmenopausal osteoporosis. J Clin Endocrinol Metab 103(7):2498-2509

5. Doyle N, Varela A, Haile S, Guldberg R, Kostenuik PJ, Ominsky MS, Smith SY, Hattersley G (2018) Abaloparatide, a novel PTH receptor agonist, increased bone mass and strength in ovariectomized cynomolgus monkeys by increasing bone formation without increasing bone resorption. Osteoporos Int 29(3):685-697

6. Varela A, Chouinard L, Lesage E, Smith SY, Hattersley G (2017) One year of abaloparatide, a selective activator of the PTH1 receptor, increased bone formation and bone mass in osteopenic ovariectomized rats without increasing bone resorption. J Bone Miner Res 32(1):24-33

7. Hattersley G, Dean T, Corbin BA, Bahar H, Gardella TJ (2016) Binding selectivity of abaloparatide for PTH-type-1-receptor conformations and effects on downstream signaling. Endocrinology 157(1):141-149

8. Miller PD, Hattersley G, Riis BJ, Williams GC, Lau E, Russo LA, Alexandersen P, Zerbini CA, Hu MY, Harris AG, Fitzpatrick LA, Cosman F, Christiansen C, ACTIVE Study Investigators (2016) Effect of abaloparatide vs placebo on new vertebral fractures in postmenopausal women with osteoporosis: a randomized clinical trial. JAMA 316(7):722-733

9. Neer RM, Arnaud CD, Zanchetta JR, Prince R, Gaich GA, Reginster JY, Hodsman AB, Eriksen EF, Ish-Shalom S, Genant HK, Wang O, Mitlak BH (2001) Effect of parathyroid hormone (1-34) on fractures and bone mineral density in postmenopausal women with osteoporosis. N Engl J Med 344(19):1434-1441

10. Forteo [package insert] (2013) Indianapolis, IN: Lilly USA, LLC; https://www.accessdata.fda.gov/drugsatfda_docs/label/2018/ 208743s0031bl.pdf Accessed June 10, 2019

11. Tymlos [package insert] (2018) Waltham, MA: Radius Health, Inc; https://www.accessdata.fda.gov/drugsatfda_docs/label/2018/ 208743s003lbl.pdf Accessed June 10, 2019

12. Prolia [package insert] (2017) Thousand Oaks, California. Amgen Inc, https://www.accessdata.fda.gov/drugsatfda_docs/label/2017/ 125320s181lbl.pdf Accessed January 24, 2020

13. Foster SA, Foley KA, Meadows ES, Johnston JA, Wang S, Pohl GM, Long SR (2008) Characteristics of patients initiating teriparatide for the treatment of osteoporosis. Osteoporos Int 19(3):373-377
14. Boytsov N, Yu M, Sugihara T, Swindle R (2015) Defining the teriparatide patient: osteoporosis sequence of care and healthcare resources. Am J Pharm Benefits 7(1):16-23

15. Mortimer K, Starzyk K, Brecht T, Cerf S, Ballentine P, Gliklich R (2019) Availability of disease activity measures from systemic lupus erythematosus patients in a large, Representative US-Based Real World Registry Cohort. Poster presented at: ICPE Annual Conference, Philadelphia, PA

16. Bandaria J, Boussios C, Donadio G, Li F, Mortimer K, Gliklich R, Starzyk K (2019) Findings from a non-alcoholic steatohepatitis (Nash) cohort developed via artificial intelligence in a large representative population in the U.S. Poster presented at: DDW Annual Conference, San Diego, CA

17. Su Z, Brecht T, Gliklich R, Menon VA (2017) Simple predictive score for pre-admission identification of risk of 30-day hospital readmission or death in heart failure. J Am Coll Cardiol 69(11):772

18. Deyo RA, Cherkin DC, Ciol MA (1992) Adapting a clinical comorbidity index for use with ICD-9-CM administrative databases. J Clin Epidemiol 45(6):613-619

19. Weiskopf NG, Hripcsak G, Swaminathan S, Weng C (2013) Defining and measuring completeness of electronic health records for secondary use. J Biomed Inform 46(5):830-836

20. Ban JK, Hao BB, McCarthy L, Guilcher SJT, Cadarette SM (2019) Denosumab utilization among older adults in Ontario: patient characteristics, persistence with therapy, and return to therapy after an extended gap. Osteoporos Int 30(9):1865-1872

21. Curtis JR, Daigle SG, Williams SA, Weiss RJ, Wang Y, Arora T (2019) Characterization of Medicare patients with a fragility fracture. Poster presented at: AMCP Managed Care and Specialty Pharmacy Annual Meeting, San Diego, CA; March 25-29

22. Chastek B, Sundquist K, Williams SA, Barrera-Sierra S, Leader D, Weiss R, Wang Y, Curtis JR (2018) Characterization of US patients with a fragility fracture. Poster presented at: National Conference for Nurse Practitioners, Lake Buena Vista, FL; May 9-12

23. Modi A, Siris S, Yang X, Fan CP, Sajjan S (2015) Association between gastrointestinal events and persistence with osteoporosis therapy: analysis of administrative claims of a U.S. managed care population. J Manag Care Spec Pharm 21(6):499-506

24. Office of the Surgeon General (US) (2004) Bone health and osteoporosis: a report of the Surgeon General. https://www.ncbi.nlm.nih. gov/books/NBK45513/ Published 2004. Accessed June 10, 2019

25. Lewiecki EM, Weiss RJ, Zapalowski C, Wang Y, de Courcy J, Williams N, Leader D, William SA (2018) Physician perception of osteoporosis medications: a US cross-sectional survey. AAFP Family Medicine Experience (FMX) Meeting, New Orleans

26. Zanchetta MB, Boailchuk J, Massari F, Silveira F, Bogado C, Zanchetta JR (2018) Significant bone loss after stopping longterm denosumab treatment: a post FREEDOM study. Osteoporos Int 29(1):41-47

27. Imel EA, Eckert G, Modi A, Li Z, Martin J, de Papp A, Allen K, Johnston CC, Hui SL, Liu Z (2016) Proportion of osteoporotic women remaining at risk for fracture despite adherence to oral bisphosphonates. Bone 83:267-275

28. Gillespie CW, Morin PE (2017) Osteoporosis-related health services utilization following first hip fracture among a cohort of privately-insured women in the United States, 2008-2014: an observational study. JBMR 32(5):1052-1061

29. Haas AV, LeBoff MS (2018) Osteoanabolic agents for osteoporosis. J Endocr Soc 2(8):922-932

Publisher's note Springer Nature remains neutral with regard to jurisdictional claims in published maps and institutional affiliations. 